\title{
Chemotherapy-related neurotoxicity in pediatric cancer patients: magnetic resonance imaging and clinical correlation
}

\author{
Mo'mena Abdel Aziz Abdou ${ }^{1 *}$ D , Hassan Ali El Kiki ${ }^{2}$, Youssef Madney ${ }^{3}$ and Ayda Aly Youssef ${ }^{1}$
}

\begin{abstract}
Background: Cancer is the second most common cause of death among children aged 1-14 years in the USA. Pediatric malignancies have elevated morbidity and mortality in the absence of proper treatment. Intensive treatment regimens have resulted in a significant increase in the number of survivors but also have been associated with the risk of developing neurotoxicity. The purpose of this study is to emphasize the role of advanced MRI techniques in the early detection of different chemotherapy neurotoxicities and make radiologists aware of them providing early management to prevent permanent damage.

Results: We evaluated 63 patients (43 males and 20 females), and their ages ranged from ( 2 to 17 years) with suspected chemotherapy-related neurotoxicity. MR examinations were performed with 1.5-T Philips systems. Clinical data were correlated with magnetic resonance imaging (MRI), and different treatment complications were diagnosed. All of our 63 patients were receiving chemotherapy treatment, and they developed different neurological symptoms. Patients diagnosed as posterior reversible encephalopathy syndrome were 41 with 8 patients had typical and 33 had atypical criteria, 16 patients diagnosed as cerebral venous sinus thrombosis with magnetic resonance venography (MRV) are the most important sequence that successfully diagnosed them, and finally, 6 patients diagnosed as methotrexate neurotoxicity with diffusion-weighted images (DWI) are the most important sequence for early diagnosis.

Conclusion: Chemotherapy is associated with certain neurotoxicities, conventional MRI can detect them, but by the use of advanced MRI techniques including MRV and DWI early detection of these neurotoxicities can occur. Therefore, the combination of conventional MRI together with advanced techniques improves the diagnostic efficacy of MRI in the early diagnosis of neurotoxicity.
\end{abstract}

Keywords: Neurotoxicity, Pediatrics, Cancer, MRI

\section{Background}

Cancer is considered the second most common cause of death among children aged 1-14 years in the USA, preceded only by accidents [1]. Cancers that are most common in children are acute lymphoblastic leukemia (ALL) (26\%), brain and central nervous system (CNS) tumors

\footnotetext{
*Correspondence: arwa730@yahoo.com

1 Department of Diagnostic and Interventional Radiology, National Cancer Institute, Cairo University, 1 Kasr Elainy Street Fom Elkalig, Cairo, Egypt

Full list of author information is available at the end of the article
}

(21\%), neuroblastoma (7\%) and non-Hodgkin lymphoma (NHL) (6\%) [2].

Current treatment modalities of cancer including systemic therapy (i.e., combination chemotherapy) and specific CNS prophylaxis (i.e., intrathecal chemotherapy with or without cranial irradiation) are potentially toxic to the nervous tissue [3]. The main chemotherapeutic agents associated with central nervous system toxic effects are methotrexate, cytarabine, vincristine, asparaginase and corticosteroids [4].

In comparison with the general population, survivors of childhood cancer have increased mortality that is due 
to several causes, and among these causes are iatrogenic risk and treatment-related side effects [5]. Treatmentrelated neurotoxicity is a potentially life-threatening clinical condition that can be challenging in diagnosis [4]. It can be difficult to differentiate between therapyassociated brain injury and recurrent disease, and it is important to immediately recognize the neurotoxicity to provide correct therapeutic management and ensuring damage reversibility [4]. Cytotoxic chemotherapies and targeted cancer therapies are known to induce early and delayed neurological toxicities; however, it is often difficult to determine which drug is causing the symptoms in patients treated with multidrug regimens [6].

The most common chemotherapy-induced neurotoxic syndromes are acute encephalopathies and posterior reversible encephalopathies (PRES) [7]. Other complications from chemotherapy treatment include progressive multifocal leukoencephalopathy, vascular complications (venous sinus thrombosis, ischemic strokes and hemorrhage), cerebellar dysfunction, spinal cord toxicity of intrathecal chemotherapy, special drugs with special presentations (methotrexate toxicity), mineralizing microangiopathy, oncologic therapy-related central nervous system (CNS) infections and finally chronic complications including mild and severe cognitive dysfunction [6].

So, this study aimed to provide early detection of different chemotherapy neurotoxicities by the use of advanced MRI techniques as MRV and DWI together with conventional MRI and makes the radiologists aware of them providing early management to prevent permanent damage.

\section{Methods}

This prospective study was approved by the local ethics committee, and informed consent had been received from parents of all patients.

\section{Patient criteria}

This study included sixty-three patients (43 males and 20 females) who were sent from the pediatric oncology department and emergency department, their ages at the time of the study ranged from (2 to 17 years) with a mean age of 8.5 years. All the patients presented to our radiology department in our hospital from 2016 to 2019.

- Inclusion criteria: pediatric age group (1-18 years), confirmed cases of cancer receiving chemotherapy, patients with clinical findings suggesting chemotherapy neurotoxicity.

- Exclusion criteria: patients with a bad general condition as some patients with disturbed conscious level (DCL) as well as those with contraindications to MRI.
The study included 60 patients who had hematological malignancies (36 patients had leukemia and 24 had lymphoma), and the other 3 patients had solid malignancies including: (adnexal yolk sac tumor, colon cancer and neuroblastoma). All of these patients were receiving chemotherapy treatment. Intravenous (IV) vincristine, intrathecal (ITH) methotrexate, corticosteroids, Ara C and IV doxorubicin were the most frequently administered chemotherapeutic agents. L-asparaginase is used in 13 patients out of 16 that were diagnosed as cerebral venous sinus thrombosis (CVST).

\section{Imaging}

All patients were submitted to the following MR sequences utilizing a 1.5-Tesla Philips Medical Systems scanner.

Pre- and post-contrast T1 weighted spin-echo images (except for 2 patients who did not receive IV contrast due to renal failure), T2 weighted spin-echo images, axial FLAIR (fluid-attenuated inversion recovery) images, axial DWI (diffusion-weighted images) at $p$ values $=0$ and $1000 \mathrm{~s} / \mathrm{mm}^{2}$ in axial orientation and ADC (apparent diffusion coefficient), MRV (magnetic resonance venography) using phase-contrast technique and T2* GRE (gradient echo) images.

For analysis, all images were transferred to a workstation using the Digital Imaging and Communications in Medicine (DICOM) format. All cases are reviewed by two expert radiologists with 7 and 15 years of experience (M. A and A.Y, respectively).

\section{Imaging evaluation and data analysis}

Patients diagnosed as PRES when there is cortical and/or subcortical vasogenic edema in typical or atypical location [8] and are classified to typical versus atypical PRES: We considered that the typical locations are the occipital and parietal lobes and the atypical locations are the frontal and temporal lobes, basal ganglia, brainstem and deep white matter [8]. Areas of restricted diffusion, contrast enhancement and hemorrhage are considered atypical presentations of PRES [9].

Grade of PRES (extension of the edema) where PRES is divided according to T2 and FLAIR signal intensity of the lesion into three grades [10].

Grade I (mild): There is cortical or subcortical white matter edema without mass effect, grade II (moderate): There is confluent edema extending from the cortex to the deep white matter without extension to the ventricular margin, grade III (severe): There is confluent edema extending from the cortex to the ventricle or edema causing midline shift or herniation. 
DWI analysis (qualitative analysis): This was done by studying the signal intensity of PRES lesions on both the DWIs and the ADC map.

For patients diagnosed as deep venous sinus thrombosis:

Our patients are classified into two groups according to the time between clinical presentation and date of MRI study [11]:

Acute stage: less than $48 \mathrm{~h}$ and sub-acute stage: $48 \mathrm{~h}-30$ days.

The affected sinus was evaluated at all pulse sequences, and the abnormality was classified as not detected, hardly detected and easily detected. Associated complications as venous infarction are evaluated also in all pulse sequences. Patients with follow-up MRI were evaluated at all pulse sequences, and the best sequence that detects the thrombosis was mentioned.

For patients diagnosed as methotrexate neurotoxicity:

Patients with clinical suspicion of methotrexate neurotoxicity, the case considered as a positive when DWI show centrum semiovale bright signal (restricted diffusion) and FLAIR show abnormal bright signals. The intensity of signal on FLAIR sequence where they are classified into negative (no abnormal signal), equivocal (slight abnormal signal detected retrograde after detection of the lesions in DWI that is hardly detected at initial assessment) and positive (abnormal high signal easily detected).

\section{Statistical analysis}

Data were coded and entered into the Statistical Package for Social Science, version 25 (SPSS), to be statistically analyzed. Quantitative variables were described as mean $\pm \mathrm{SD}$ and median. Qualitative variables were described as frequency and percent and compared using Chi-square test were $\mathrm{p}$-value of significant difference if less than 0.01 .

\section{Results}

This prospective study included sixty-three patients, 43 (68\%) were males and 20 (32\%) were females. Their ages at the time of the study ranged from 2 to 17 years.

According to the clinical presentation MRI was performed on 63 patients, and the following conditions were diagnosed from the obtained MR images as shown in Table 1 which summarize different clinical presentations, the causative chemotherapy and the imaging findings. All patients were receiving chemotherapy treatment.

\section{Results for PRES}

In this study, the 41 patients diagnosed as PRES showed that hematological cancers are the commonest primary cancers representing $92.7 \%(N=38)$ of the cases and leukemia was the most common cancer among them $51.2 \%$ $(N=21)$ as demonstrated in Fig. 1.

\section{Initial MRI}

All patients with suspected PRES underwent MRI with a time interval between MRI and the clinical event ranges from the same day to 10 days.

\section{According to the location of signal abnormality}

The occipital lobe was the most commonly affected lobe, and its involvement was seen in $87.8 \%(N=36)$ of the patients, followed by the parietal lobe $85.4 \%$

Table 1 Summary of different clinical presentations, the causative chemotherapy and the imaging findings

\begin{tabular}{|c|c|c|c|}
\hline & PRES (No./\%) & $\begin{array}{l}\text { Cerebral venous thrombosis } \\
\text { (No./\%) }\end{array}$ & Methotrexate neurotoxicity (No./\%) \\
\hline Number of patients & $\begin{array}{l}41(65 \%) \\
(\text { Males }=30, \text { females }=11)\end{array}$ & $\begin{array}{l}16(25 \%) \\
(\text { Males }=11, \text { females }=5)\end{array}$ & $\begin{array}{l}6(10 \%) \\
(\text { Males }=2, \text { females }=4)\end{array}$ \\
\hline Clinical picture & $\begin{array}{l}\text { Seizures (34) } \\
\text { Headache (7) } \\
\text { Consciousness alteration (6) } \\
\text { Visual abnormality (6) } \\
\text { Others clinical manifestation (12) }\end{array}$ & $\begin{array}{l}\text { Headache (12) } \\
\text { Seizures (4) } \\
\text { Loss of consciousness (2) } \\
\text { Visual abnormalities (1) }\end{array}$ & $\begin{array}{l}\text { Seizures ( } 3 \text { ) } \\
\text { altered mental status (1) } \\
\text { Left-sided facial droop and slurred } \\
\text { speech (1) } \\
\text { One-sided weakness (1) }\end{array}$ \\
\hline $\begin{array}{l}\text { The percentage of patients } \\
\text { using this type of chemo- } \\
\text { therapy }\end{array}$ & $\begin{array}{l}\text { IV vincristine (87.8\%) } \\
\text { Intrathecal (ITH) methotrexate, Ara C, } \\
\text { hydrocortisone }(87.8 \%) \\
\text { IV doxorubicin }(51.2 \%) \\
\text { Oral prednisone }(90.2 \%) \\
\text { IV L-asparaginase }(46.3 \%) \\
\text { IV cyclophosphamide }(46.3 \%) \\
\text { IV bleomycin, vinblastine, dacar- } \\
\text { bazine, oxaliplatin, cisplatin }(2.4 \%)\end{array}$ & $\begin{array}{l}\text { IV vincristine (100\%) } \\
\text { Intrathecal (ITH) methotrexate, Ara C, } \\
\text { hydrocortisone (100\%) } \\
\text { IV doxorubicin (100\%) } \\
\text { Oral prednisone }(100 \%) \\
\text { IV L-asparaginase }(81.3 \%) \\
\text { IV methotrexate }(50 \%) \\
\text { IV cyclophosphamide }(37.5 \%) \\
\text { Oral } 6 \text { mercaptopurine }(12.5 \%)\end{array}$ & $\begin{array}{l}\text { All these patients are receiving } \\
\text { intrathecal methotrexate, and the time } \\
\text { between the intrathecal methotrex- } \\
\text { ate and the occurrence of symptoms } \\
\text { ranged from } 6 \text { to } 13 \text { days }\end{array}$ \\
\hline Imaging findings & $\begin{array}{l}\text { Hyperintense signal on } \mathrm{T} 2 \mathrm{WI} \text { and } \\
\text { FLAIR images and iso to hypointense } \\
\text { signal on T1WI }\end{array}$ & $\begin{array}{l}\text { Non-visualization of occluded veins } \\
\text { or sinuses due to absent signal and } \\
\text { flow defects }\end{array}$ & $\begin{array}{l}\text { Restriction in diffusion-weighted } \\
\text { images (bright signal) in centrum } \\
\text { semiovale }\end{array}$ \\
\hline
\end{tabular}




\section{Frequency of the primary cancer in PRES}

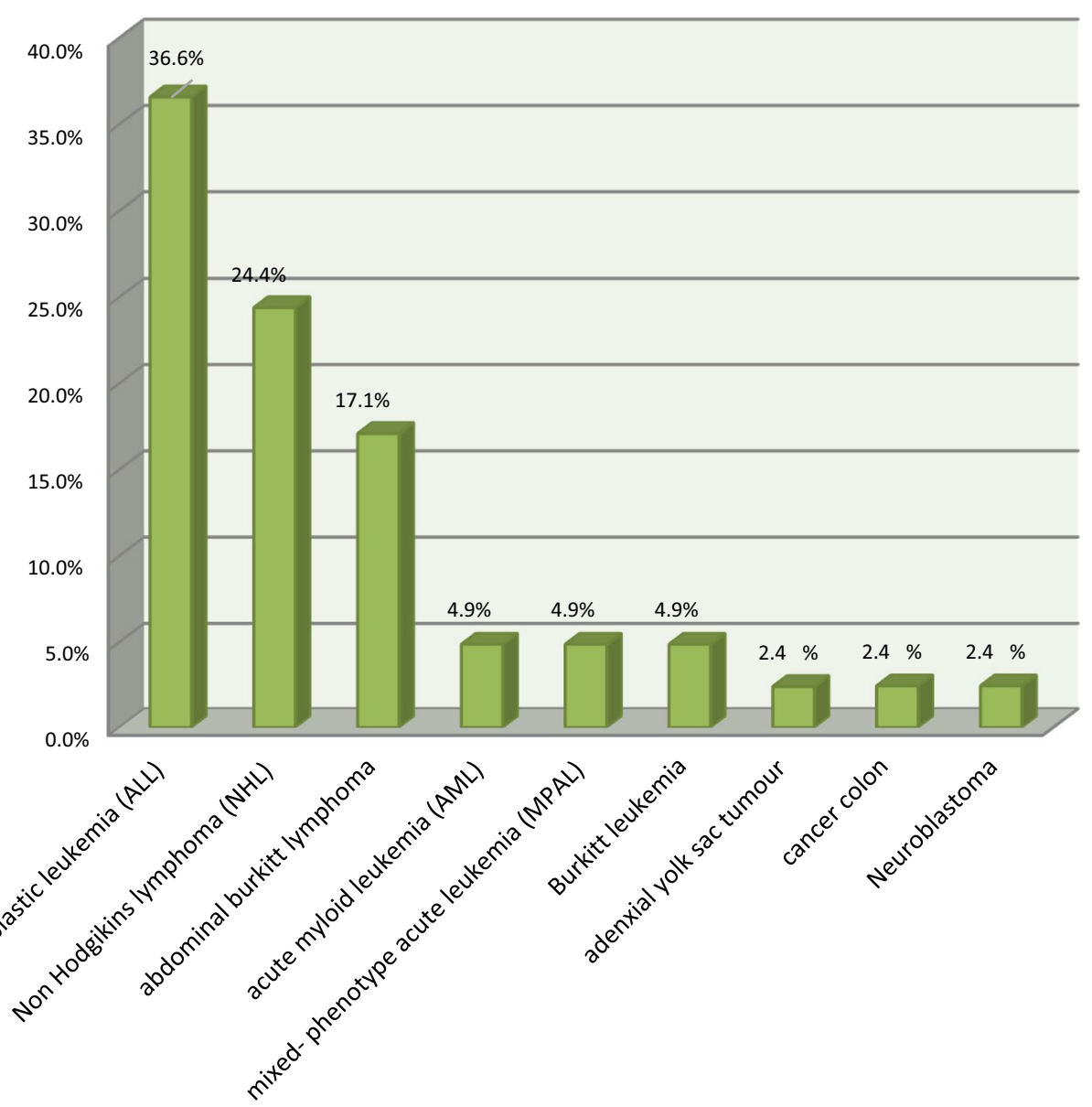

Fig. 1 Frequency of the primary cancer in PRES

$(N=35)$, followed by frontal (34.1\%), temporal (31.7\%), cerebellum(31.7\%) and other regions as thalami, brain stem and corpus callosum with predominant involvement of the cerebral cortex seen in $61 \%(N=25)$, subcortical predominant involvement was seen in $22 \%$ $(N=9)$, and involvement of both of them was seen in rest of patients.

Lesions were seen bilateral in 92.7\% $(N=38)$ of patients, unilateral in the rest of patients. They also showed an asymmetrical distribution in $80.5 \%(N=33)$.

Grading of disease according to the extension of edema was done using the T2/FLAIR images:

The most common form of the disease was grade II representing $65.9 \%(N=27)$ followed by grade I seen in $29.2 \%(N=12)$ and grade III in $4.9 \%(N=2)$ of the patients (Figs. 2 and 3).

Out of the 27 patients with grade II (moderate form) 1 has an adverse outcome, 7 died and 19 patients resolved. Out of the 12 patients with grade I (mild form) 4 have adverse outcomes, 6 resolved and 2 died. The 2 patients with grade III (severe form) of the disease died. This study shows that there was a statistically significant difference found between grades II and III ( $p$ value $=0.01$ ) in the clinical outcome of the patients.

Out of the 41 patients, $19.5 \%(N=8)$ patients demonstrated typical MRI findings, and $80.5 \%(N=33)$ patients demonstrated atypical findings. Of the 8 patients with typical PRES (group A), 75\% $(N=6)$ resolved and $25 \%(N=2)$ had an adverse outcome. While the 33 patients with atypical PRES (group B), $51.5 \%(N=17)$ of them resolved and $48.5 \%(N=16)$ had an adverse outcome. There was no statistically significant difference between the two groups in the outcome of the disease $(p$ value $=0.69)$. 


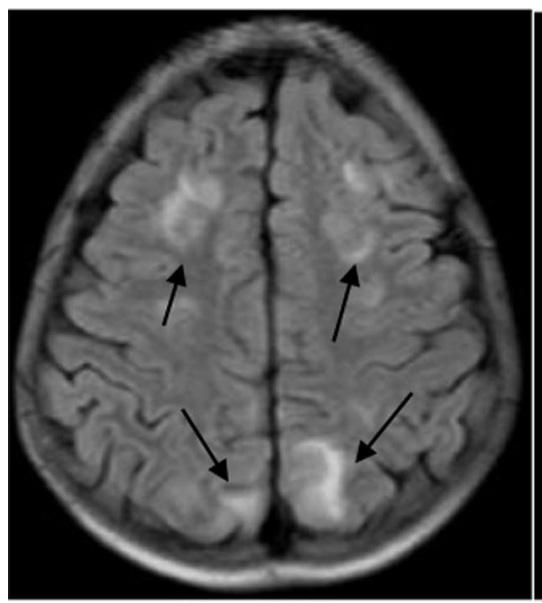

a

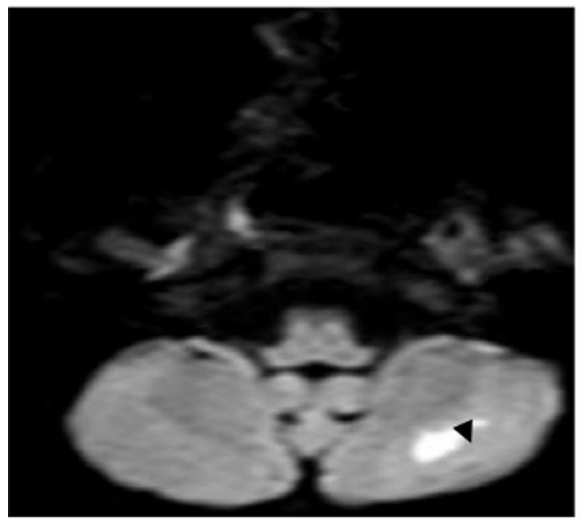

d

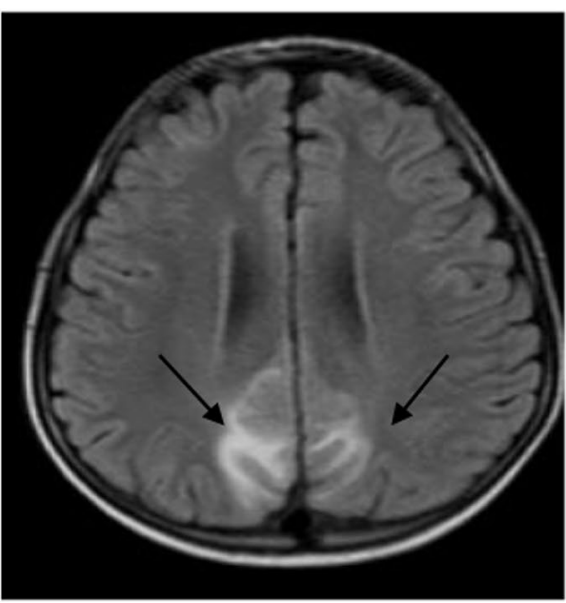

b

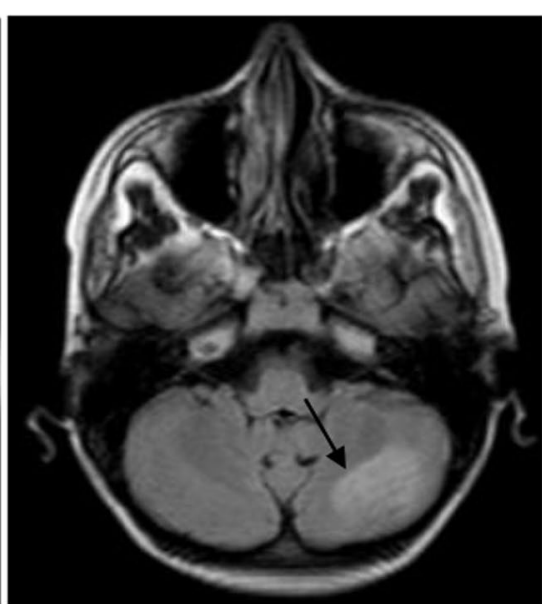

C

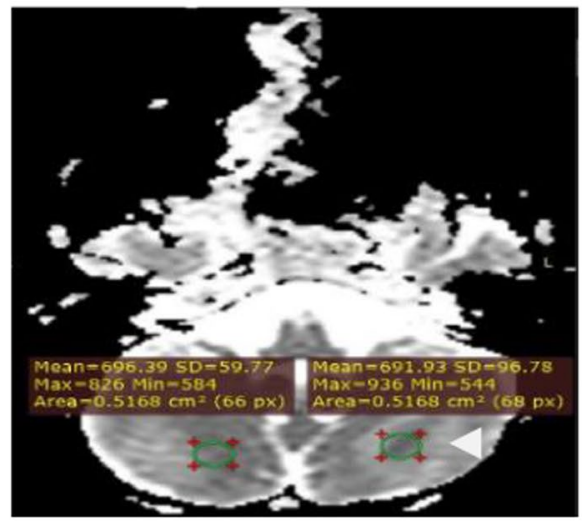

e

Fig. 2 A 9-year-old male known leukemic patient was on day 11 of his chemotherapy regimen (IV vincristine, IV doxorubicin, IV L-asparaginase, oral prednisone and intrathecal methotrexate, hydrocortisone, ARA C) when he presented with headache and convulsions. Brain MRI was done on the same day of clinical presentation. MRI revealed: FLAIR (a-c) showed bilateral almost symmetrical (except at cerebellum it is asymmetrical) predominantly subcortical areas of abnormally high signal intensity are seen in the occipital lobe (b), frontal (a), parietal lobes (a) and left cerebellar hemisphere (black arrows). No sizable areas of hemorrhage were seen. DWI (d): The lesion in the left cerebellar hemisphere showed high signal (black arrowhead). ADC map (e): The lesions show isointense signal compared to the contralateral normal brain parenchyma denoting pseudonormalization phenomenon (white arrowhead). There was no appreciable contrast enhancement (not shown). The MRI findings are consistent with an atypical MRI pattern of PRES with grade II edema extension. Follow-up MRI study of the brain after 2 months which showed complete resolution of all lesions

\section{Atypical MRI findings}

The atypical PRES classified into atypical location and atypical presentation. The atypical presentation was seen in the form of contrast enhancement which was seen in $7 \%(N=3)$ of the patients in the form of leptomeningeal enhancement, hemorrhage in $2.5 \%(N=1)$ of the patients in the form of hematoma and restricted diffusion in $34 \%$ $(N=14)$ of the patients.

Among the 14 patients who had restricted diffusion, 7 patients show clinical resolution, 4 show neurological deficits, and 3 patients show death (from different causes as renal failure, intestinal obstruction, hypotension and breathing problems). We found that there was no statistically significant difference between patients diagnosed as PRES with areas of diffusion restriction and those who had no diffusion restriction in the clinical outcome of the patients $(p$ value $=0.2238)$.

\section{Follow-up MRI}

Out of 41 patients diagnosed as PRES, 63.5\% $(N=26)$ did follow-up MRI with a time interval between the initial MRI and the first follow-up MRI ranges from 3 weeks to 2 months and the results of follow-up MRI are shown in Fig. 4. 


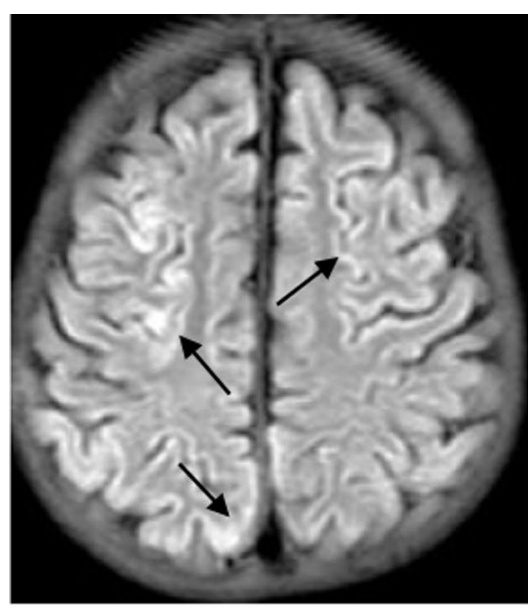

a

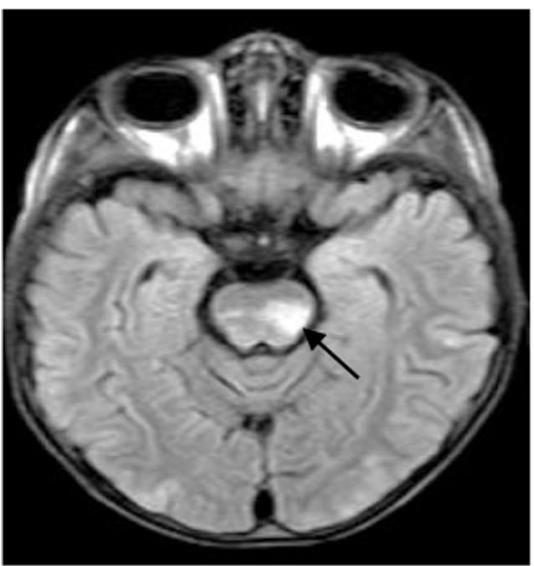

d

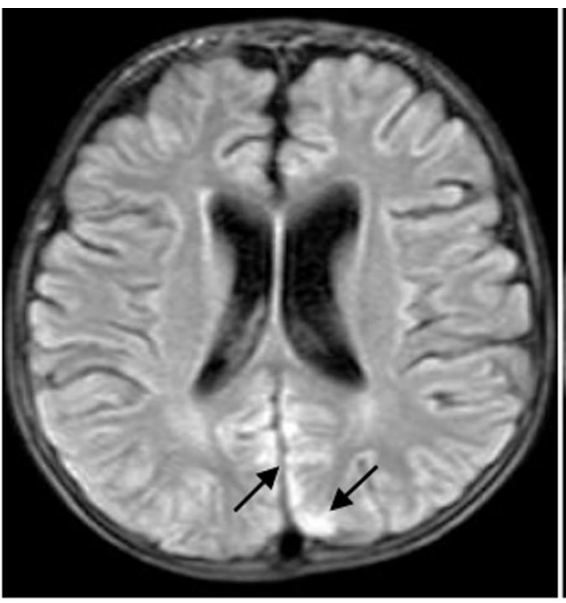

$\mathrm{b}$

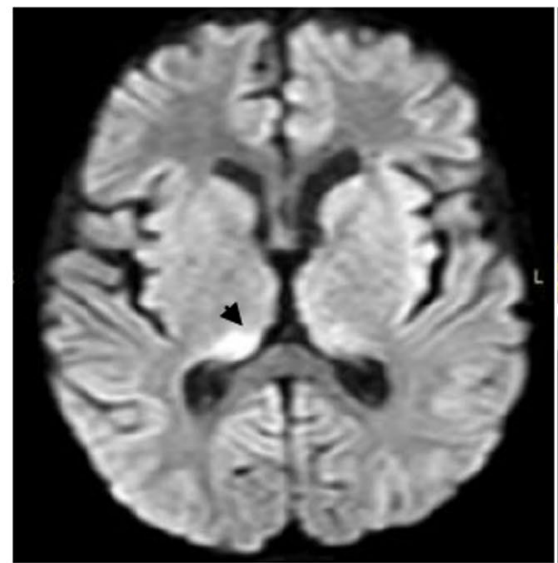

e

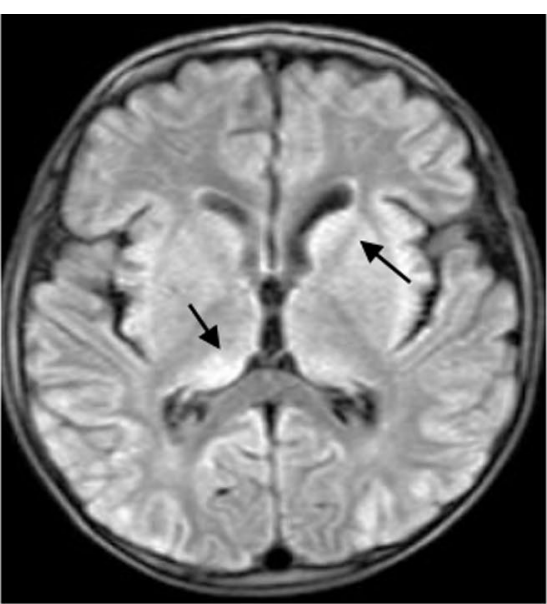

C

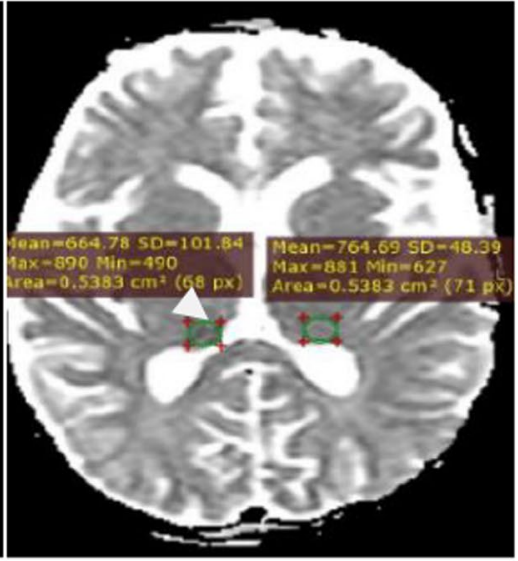

$f$

Fig. 3 An 8-year-old male diagnosed as lymphoma. The patient received a course of chemotherapy (IV vincristine, IV cyclophosphamide, oral prednisone and intrathecal methotrexate, hydrocortisone, ARA C) after which he developed a disturbed conscious level. Brain MRI was done after 1 day of clinical presentation. MRI revealed: FLAIR images (a-d) show bilateral asymmetrical cortical areas of abnormal high signal intensity are seen in frontal (a), parietal (a) and occipital lobes (b) (black arrows). Also, there was bilateral asymmetrical affection of the thalami (c) and involvement of brain stem (d) and basal ganglia (c) (black arrows). DWI (e): The lesions in thalami show high signal (black arrowhead). ADC map (f): The lesions show low signal compared to the contralateral normal brain parenchyma denoting restriction (white arrowhead). There was no appreciable contrast enhancement of the lesions (not shown).The MRI findings are consistent with an atypical MRI pattern of PRES with grade I edema extension of the cerebral lesions and thalami and grade II edema of the brain stem. The patient died after 1 week

\section{Results for cerebral venous sinus thrombosis (CVST)}

In this study, all patients diagnosed as CVST (16 patients) have hematological cancers and the most common cancer among them was leukemia $68.7 \%(N=11)$ including ALL (56.2\%) and mixed-phenotype acute leukemia (12.5\%), and other types of cancers include NHL (18.8\%) and lymphoblastic lymphoma (12.5\%).

\section{Initial MRI}

All patients with suspected CVST underwent MRI with the time interval between the clinical onset and date of MRI ranges from the same day to 2 days after clinical symptoms. More than one occluded sinus could be found in some patients. A total of 39 thrombosed venous sinuses were identified in our 16 patients. No patient had isolated cortical vein thrombosis without dural sinus involvement. According to the affected venous sinus or veins, the transverse sinuses are the most commonly affected sinuses $75 \%(N=12)$ as shown in Table 2 .

Our patients are classified into two groups: acute stage: less than $48 \mathrm{~h}(N=10,62.5 \%)$ and sub-acute stage: $48 \mathrm{~h}-30$ days $(N=6,37.5 \%)$. The main magnetic 


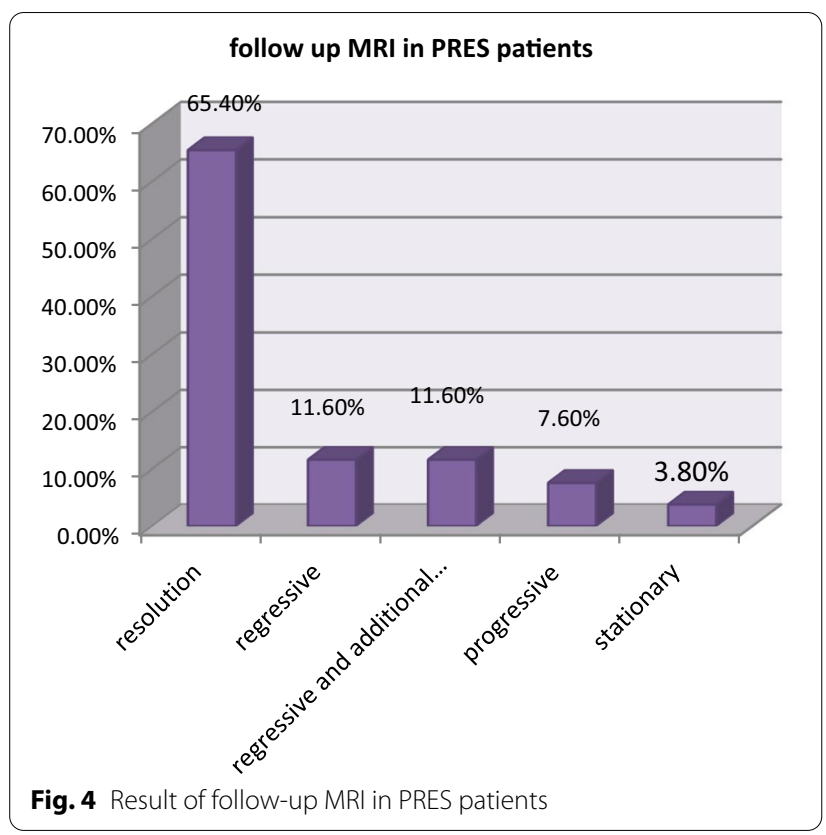

resonance venography (MRV) findings in this study were the non-visualization of occluded veins or sinuses due to absent signal and flow defects. MRV successfully diagnosed all cases (Fig. 5). The best sequence that evaluates sinus thrombosis in the acute stage was 3D MRV (100\%), followed by T1 SE, T2* GRE and T1 FFE post-contrast $60 \%(N=6)$, while the best MRI sequence in the early sub-acute stage was 3D MRV (100\%), followed by T1SE post-contrast, T1 FFE post-contrast and T2* GRE 66.7\% $(N=4)$. Parenchymal changes in the patients with CVST were due to thrombosis in 3 patients and include hemorrhagic infarction (18.8\%).

\section{Follow-up MRI}

Follow-up MRI was done at a period ranging from 15 days to 3 months between initial MRI and the first follow-up MRI. It was done in $56.3 \%(N=9)$ of the patients with CVST. Out of the 9 patients who underwent follow-up MRI 18.8\% $(N=3)$ of them showed complete recanalization and $37.5 \%(N=6)$ patients showed residual thrombosis.

However, MRV successfully diagnoses all patients with CVST in the initial MRI in follow-up MRI and it is not the best sequence to detect residual thrombus (Fig. 6). We found that the best sequence in detection of residual thrombosis in a patient with follow-up MRI in the late sub-acute stage was $\mathrm{T} 1$ post-contrast SE $60 \%(N=3)$ of the patient followed by FLAIR $40 \%(N=2)$, while for those who did follow-up MRI in the chronic stage (more than 1 month) best sequence was FLAIR in $100 \%(N=5)$ of the cases.

\section{Results for methotrexate neurotoxicity}

All patients in this study diagnosed as methotrexate neurotoxicity have hematological cancers, and the most common cancer among them was leukemia $66.7 \%$ $(N=4)$, and rest of the patients have lymphoma $33.3 \%$ $(N=2)$. The most common type of leukemia was ALL $(N=3,50 \%)$.

\section{MRI evaluation}

All patients with suspected methotrexate neurotoxicity did MRI after the occurrence of clinical events at a period ranging from the same day to 2 days. All of them showed restriction in diffusion-weighted images (bright signal). While in FLAIR, three of our patients $(50 \%)$ showed equivocal findings, two showed negative findings and one is positive showing high signal in FLAIR.

According to the location of the lesions, all abnormal signals were in the centrum semiovale and bilateral. Patients who showed symmetrical distribution of the lesions represent $66.7 \%(N=4)$ and $33.3 \%(N=2)$ show asymmetrical distribution (Fig. 7).

All patients with methotrexate neurotoxicity did the first follow-up MRI (except one patient lost follow-up) within 2 weeks from the initial one which shows resolution of the diffusion abnormality with interval development of abnormal signal intensity on FLAIR and T2WI.

Table 2 location of thrombosis in cerebral venous sinuses and veins

\begin{tabular}{llcr}
\hline Sinus affected & & Number of patients affected by thrombosis & Percentage (\%) \\
\hline Superior sagittal sinus & & 11 & 68.80 \\
& Straight sinus & 1 & 6.30 \\
& Left transverse sinus & 6 & 37.50 \\
& Right transverse sinus & 6 & 37.50 \\
& Left sigmoid sinus & 4 & 25.00 \\
& Right sigmoid sinus & 4 & 25.00 \\
& Cortical veins & 7 & 43.80 \\
\hline
\end{tabular}




\section{Discussion}

Chemotherapy-related neurotoxicity is a potentially lifethreatening clinical condition that can represent a diagnostic challenge [5]. Early identification of common and less common imaging findings and differentiating diagnoses between therapy-associated brain injury and recurrent disease may point toward the correct direction and may facilitate early diagnosis and institution of appropriate treatment to reverse or at least limit the injury to the developing brain [12]. Standard neuroimaging may identify a variety of neurotoxic squeals in pediatric cancer patients, and specific neuroimaging findings frequently indicate a need to alter anticancer therapy [12].

As regards posterior reversible encephalopathy syndrome (PRES), it is a clinico-radiologic entity and it became an increasingly recognized condition in cancer patients undergoing therapy [13]. A clinical diagnosis of PRES includes the presence of headache, seizures, encephalopathy and visual disturbances, as well as radiologic findings of focal reversible vasogenic edema, best seen on MRI of the brain [13].

In this study, hematological cancers were the most common primary cancers in our PRES patients and seizures were the most common presenting symptom. The most commonly administered agents were IV vincristine, ITH (methotrexate, Ara C and hydrocortisone) and IV doxorubicin before and during the onset of PRES.

These results go with a study for Khan et al. [14] who did their study on children having cancer. They identified 37 patients with PRES among 5217 children treated during the study, and the primary tumor was leukemia representing (67.5\%), brain tumors (13.5\%) and other solid tumors (19\%). The most common presenting symptom includes seizures (97\%), and steroids use was seen in $78 \%$ of patients.

Kamiya-Matsuoka et al.s study [13] was done identifying 69 cancer patients with PRES which showed that chemotherapy intake of the patients is seen in $70 \%$ of them and the most commonly administered agents were doxorubicin and vincristine and cortisone intake was recorded in $41 \%$ of the patients.

This work found that diffusion restriction and typical and atypical MRI criteria in PRES do not affect the clinical outcome.
The study for Wagih et al. [15] included thirty-six patients diagnosed as PRES and concluded that most of the patients $(88.9 \%)$ with diffusion restriction were completely reversible.

Regarding cerebral venous sinus thrombosis (CVST), it is a neurological condition with non-specific symptoms. Magnetic resonance imaging (MRI) in conjunction with MR venography (MRV) is the most sensitive technique for the diagnosis of cerebral venous thrombosis [16]. One such predisposing condition is acute lymphoblastic leukemia (ALL) and its intensive induction chemotherapy [17]. The importance of chemotherapy in the pathogenesis of ALL-associated CVST is indicated by the observation that over $90 \%$ of cases occur during induction therapy; therefore, research has focused on chemotherapeutic agents administered and their influence on hemostasis such as asparaginase [17].

This study found that hematological cancers were the primary cancers in CVST patients, where leukemia was on the top of the list. L-asparaginase is used in (81.3\%). Also, the intake of oral cortisone was seen in $100 \%$ of the cases.

The study for Alsaid et al. [18] that did their study on a 9-year-old boy with acute lymphoblastic leukemia (ALL) treated with L-asparaginase, said that the children at greatest risk for CVST are generally those receiving L-asparaginase concomitant with prednisone, and also they said that over $90 \%$ of cases occur during induction therapy.

L-Asparaginase reduces circulating levels of many hemostatic proteins including fibrinogen, plasminogen and antithrombin-III. These anticoagulant deficiencies result in impaired inhibition of thrombin, which has been proposed as the main pathogenic mechanism for thrombosis [6].

This study concluded that the best MRI sequence that detects thrombosis in the acute stage was 3D MRV (100\%), followed by T1 SE and T2* GRE in pre-contrast sequences and T1 FFE in post-contrast, while the best MRI sequence in the early sub-acute stage was 3D MRV (100\%), followed by T2* GRE in pre-contrast sequences and T1SE and T1 FFE in post-contrast sequences.

\footnotetext{
(See figure on next page.)

Fig. 5 A 5-year-old male diagnosed as acute lymphoblastic leukemia on day 17 of his chemotherapy protocol (IV vincristine, IV doxorubicin, IV L-asparaginase, oral prednisone and intrathecal methotrexate, hydrocortisone, ARA C), he presented with convulsions, and MRI was done at the same day after the clinical presentation. MRI revealed: total thrombosis of superior sagittal sinus, cortical veins, right and left transverse sinuses as well as straight sinus showing high signal on T1 SE (a) (short black arrow), loss of signal void on T2 (b) (long black arrow), isointense signal on FLAIR (c) (short white arrow), low signal on T2* (d) (white arrowhead) and non-visualization of occluded veins or sinuses on MRV (f1, f2) (white arrows). The thrombosed sinuses are not detected on T1 post-contrast (e1, e2) (black arrowhead) and DWI and ADC images (not shown). There were also bilateral acute frontal hemorrhagic venous infarctions (black arrows in $\mathbf{d}$ )
} 

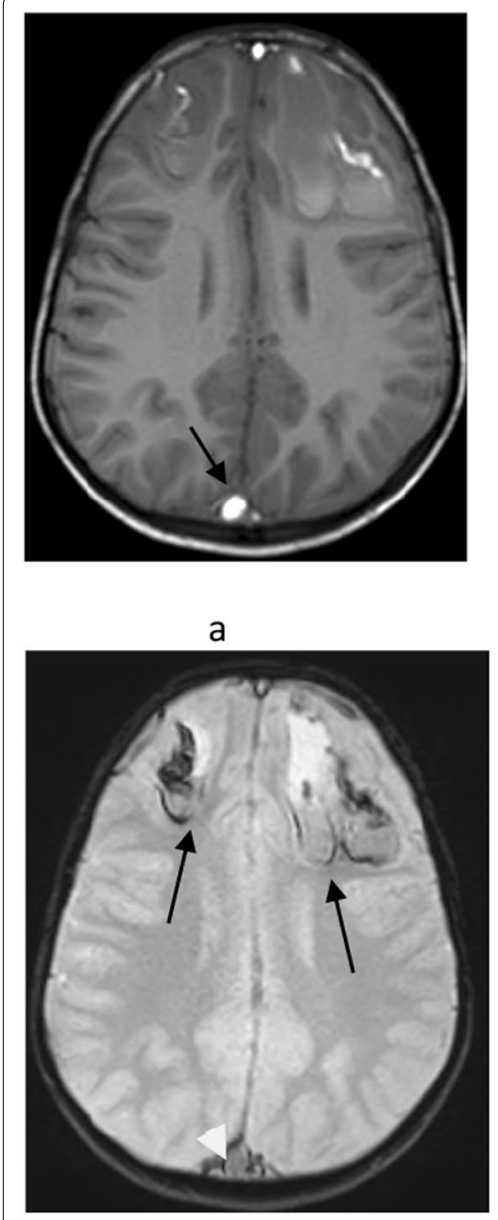

d

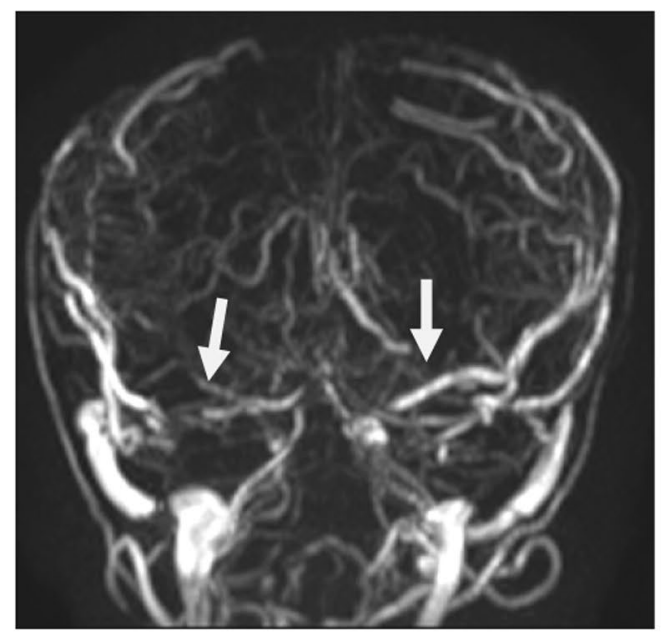

f1

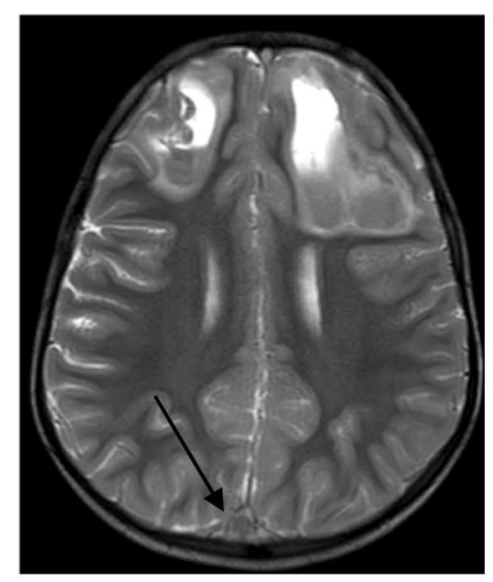

b

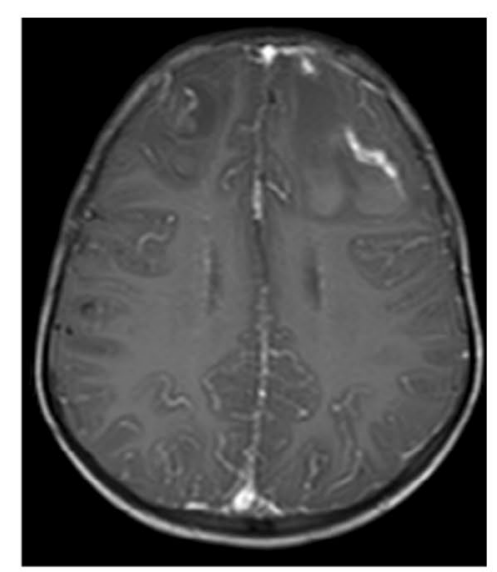

e1

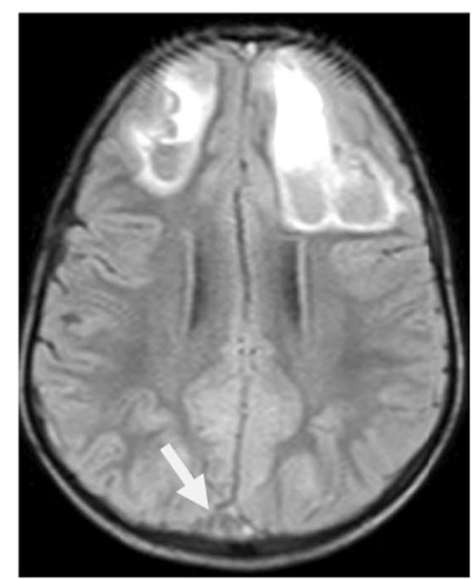

C

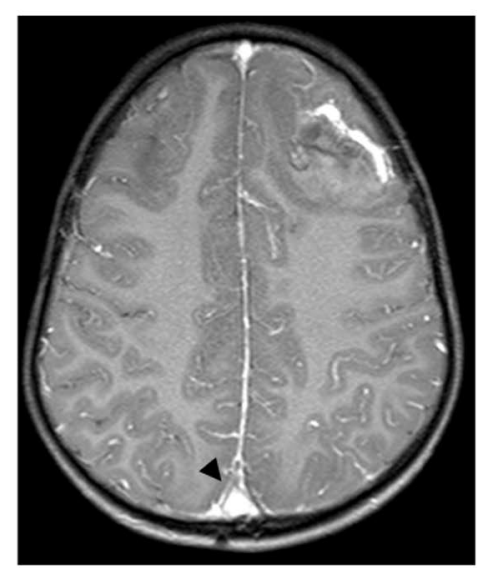

e2

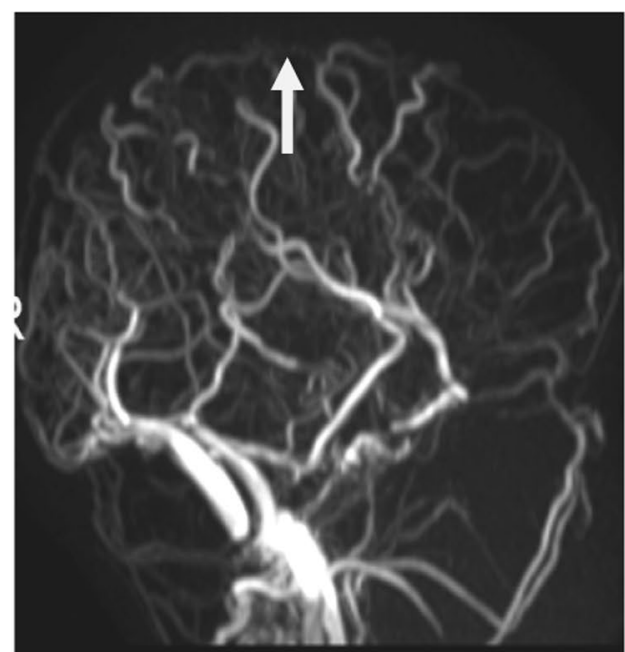

f2

Fig. 5 (See legend on previous page.) 

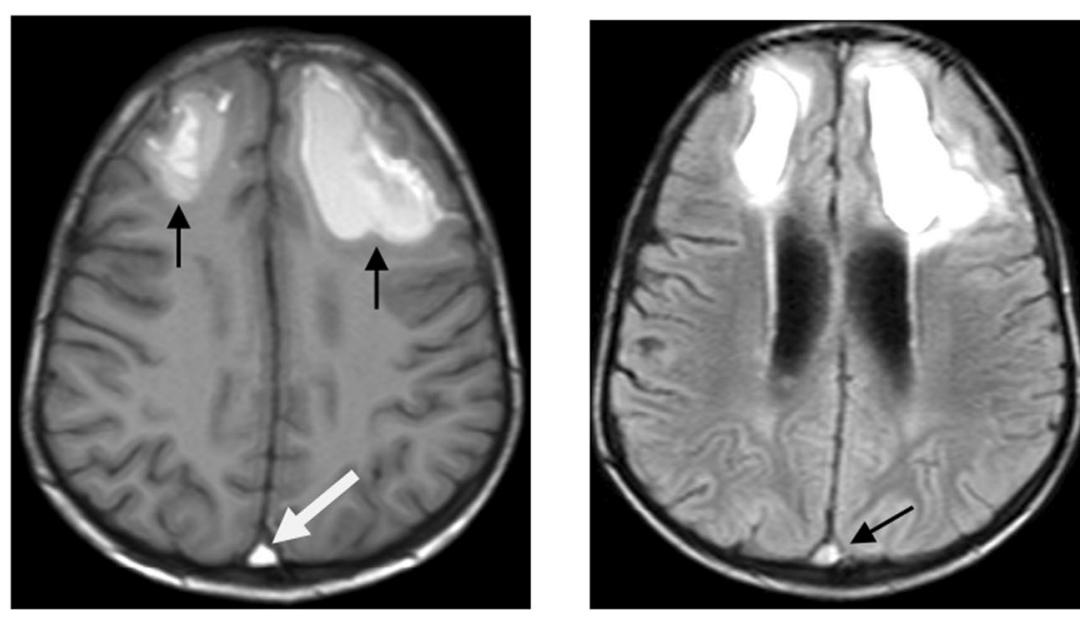

b a

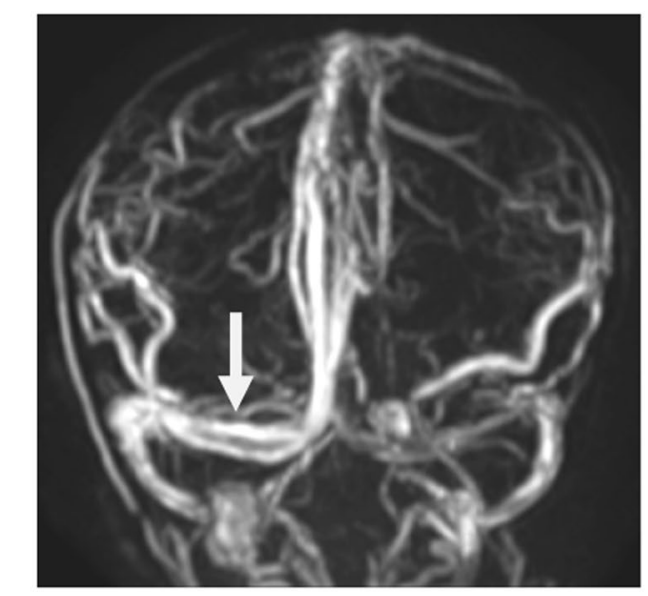

c2

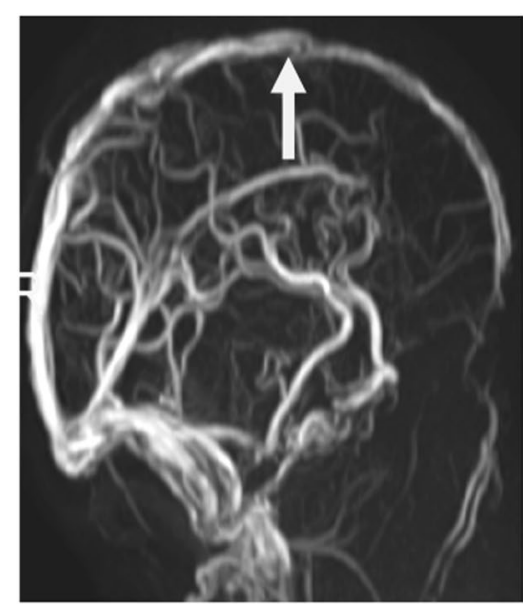

c1

Fig. 6 Follow-up of the same patient in (Fig. 5). The patient stopped chemotherapy and received clexane; follow-up MRI was done 15 days after the initial MRI (Fig. 5) and revealed partial recanalization of the thrombosed sinuses and aging of the bilateral frontal hemorrhagic venous infarctions (black arrows in $\mathbf{a}$ ) best appreciated at T1 SE (a) (white arrow), FLAIR (b) (black arrow) and partial recanalization of the thrombosed sinuses in 3D MRV (c1 and $\mathbf{c} 2$ white arrows)

El Damarawy et al. [19] concluded that MRV successfully diagnoses all patients. Hemorrhagic infarction was seen in $26.7 \%$ of the patients.

Choi et al. [20] concluded that the most sensitive MR sequence for the diagnosis of CVST was gadoliniumenhanced T1WI (82\%). GRE was the most sensitive of the non-enhanced sequences $(77.4 \%)$ at the acute stage. T1WI (69.6\%), FLAIR (34.4\%) and DWI (18.8\%) were less sensitive. Overall, the non-enhanced MR sequences showed relatively lower diagnostic performance compared to gadolinium-enhanced T1WI.

This work found that the best sequence in detection of residual thrombosis in the patients with follow-up MRI in the late sub-acute stage was T1 post-contrast
SE of the patient followed by FLAIR, while for those who did follow-up MRI in the chronic stage (more than 1 month) best sequence was FLAIR.

This goes with results for Choi et al. [20] who concluded that the diagnostic performance of GRE decreased due to the loss of the characteristic low signal due to the evolution of the thrombus. FLAIR showed increased sensitivity, as a high intense signal of CVST developed during follow-up. Gadolinium-enhanced T1WI is necessary for follow-up studies of CVST. If the patient cannot tolerate contrast agents, the use of FLAIR for the remaining CVST may be helpful.

As regards methotrexate (MTX) neurotoxicity, it occurs in about $9 \%$ of patients treated for ALL [21]. It 


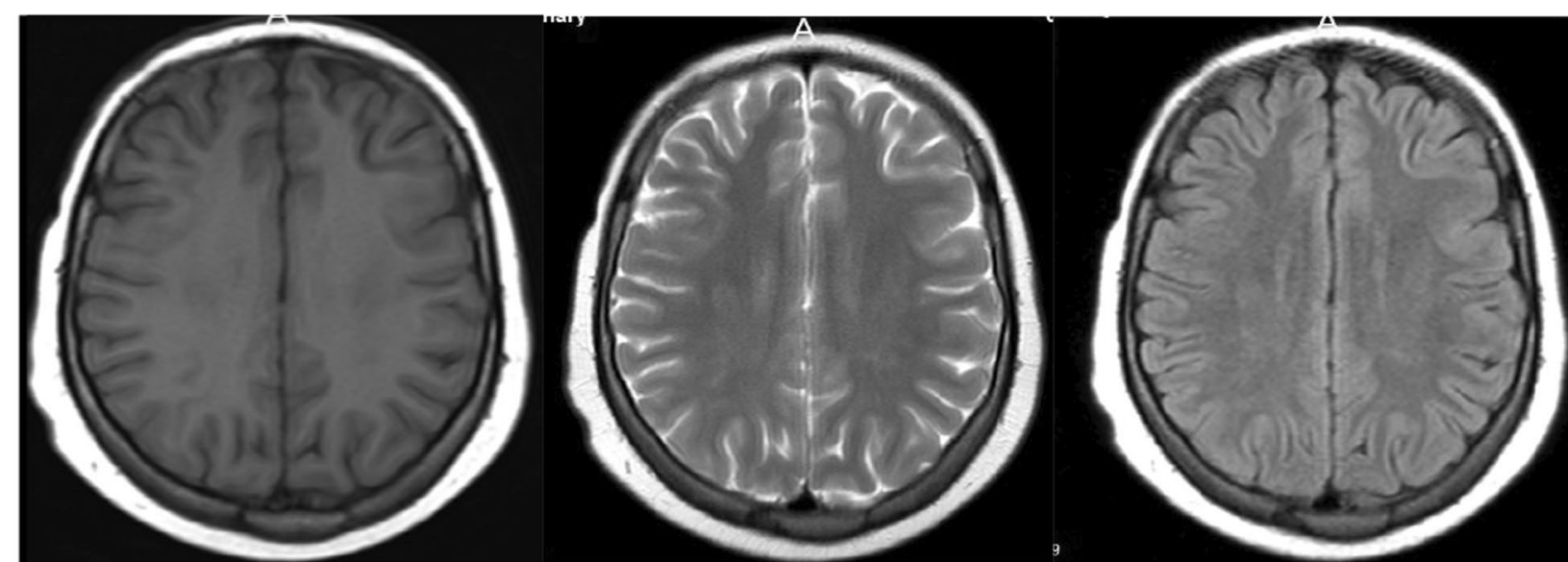

a

$\mathrm{b}$

C

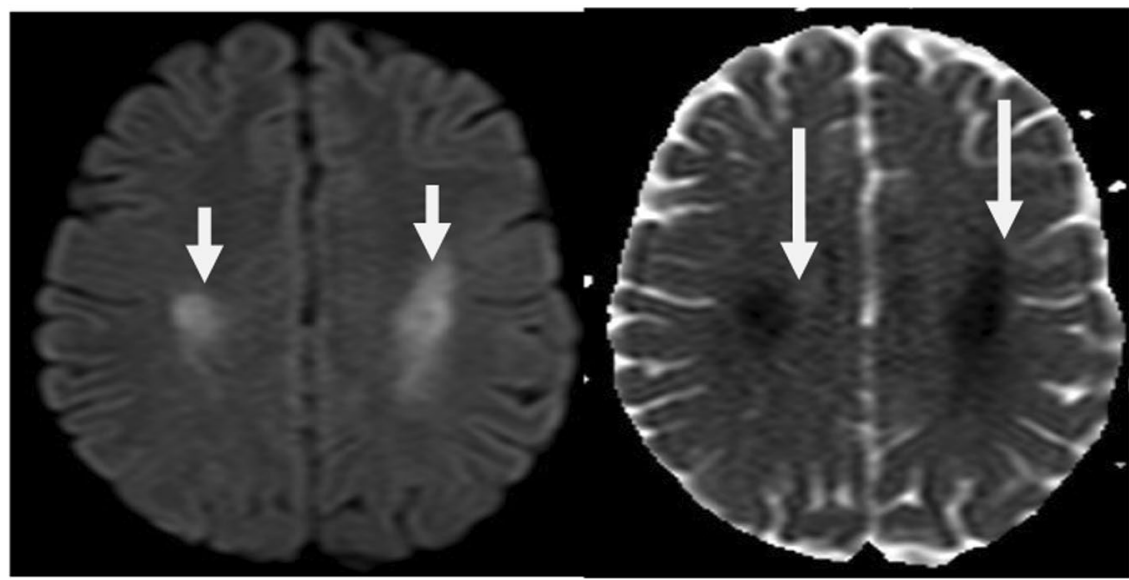

\section{d}

e

Fig. 7 A 14-year-old female known to have ALL, in her consolidation phase of chemotherapy treatment (high dose methotrexate, IV vincristine, IV cyclophosphamide, 6-mercaptopurine and intrathecal methotrexate, hydrocortisone, ARA C), she developed right-sided pain that progressed to right-side weakness involving upper and lower limbs. MRI was done 2 days after presentation. MRI revealed: bilateral symmetrical centrum semiovale areas of high signal in DWI (d) (short white arrows) and low signal in ADC (e) (long white arrows) denoting diffusion restriction, and this signal is hardly detected in T2 and FLAIR ( $\mathbf{b}$ and $\mathbf{c}$ ) images, not appreciated in all other pulse sequences as T1 (a). The MRI findings together with clinical data are consistent with methotrexate neurotoxicity

usually affects the deep white matter region leading to leukoencephalopathy, which has varying clinical manifestations ranging from acute neurological disturbances to seizures or chronic permanent encephalopathy [21].

In this study, all patients with methotrexate neurotoxicity have hematological cancers. All patients show bilateral symmetrical or asymmetrical centrum semiovale lesions. Diffusion-weighted imaging (DWI) is a rapid non-invasive MRI technique used in imaging of brain abnormalities. In this study, the initial MRI scan showed abnormal restricted diffusion in the centrum semiovale in all cases, whereas the FLAIR was positive only in one case and equivocal in three cases. In the follow-up MRI of these patients the diffusion abnormality resolved with interval development of abnormal signal intensity on FLAIR and T2WI.

This study goes with a study for Youssef et al. [22] which was done on 17 patients with MTX neurotoxicity. All patients had hematological malignancies. The MRI was done at periods ranging from the same day to 2 days. 
Youssef et al. [22] said that the MR imaging scan showed abnormal restricted diffusion in the centrum semiovale in all patients. No abnormality on the FLAIR sequence in $50 \%$ cases, equivocal in $30 \%$ of cases and show bright signal only in $20 \%$ of cases. In $50 \%$ of cases in initial studies, the abnormal diffusion was symmetrical in both centrum semiovale, and in $40 \%$ of the cases the abnormal signal was asymmetrical more on the left centrum semiovale.

This study concluded that DWI is the most important sequence in the initial MRI evaluation of patients with suspected acute methotrexate neurotoxicity. Therefore, it provides a rapid, non-invasive readily available accurate tool by which neurotoxicity can be early detected and treated.

The limitations of this study are that it was done on a small number of patients and only qualitative assessment of ADC values was done not a quantitative one, for which we recommend a larger study to be conducted taking into consideration these limitations.

\section{Conclusions}

The wide variety of CNS abnormalities that occur during and after treatment of pediatric cancer is related to the disease itself or the treatment, and it may affect the developing/maturing brain. The brain changes occur due to chemotherapy usually reversible both clinically and radiologically on withdrawing the offending drug as early as possible. Improved neuroimaging techniques help to characterize CNS abnormalities caused by either recurrent disease as well as treatment-related neurotoxicity. Therefore, the proper knowledge of chemotherapeutic side effects on CNS and their radiological appearance is important to early diagnose the problem and treat it well to ensure reversible neurological damage.

So we can conclude that however, conventional MRI may help in the diagnosis of treatment-related neurotoxicity, combination of conventional MRI together with advanced MRI techniques as MRV and DWI improve the diagnostic efficacy of MR imaging in the diagnosis of these toxicities.

\footnotetext{
Abbreviations

MRI: Magnetic resonance imaging; FLAIR: Fluid-attenuated inversion recovery; DCL: Disturbed conscious level; MRV: Magnetic resonance venography; DICOM: Digital Imaging and Communications in Medicine; SPSS: Statistical Package for Social Science; PRES: Posterior reversible encephalopathy syndrome; NHL: Non-Hodgkin lymphoma; ALL: Acute lymphoblastic leukemia; DWI: Diffusion-weighted images; CNS: Central nervous system; ADC: Apparent diffusion coefficient; CVST: Cerebral venous sinus thrombosis; IV: Intravenous; T2WI:T2 weighted images; 3D: 3 Dimensional; T1 SE:T1 spin echo; FFE: Fast field echo; ITH: Intrathecal; GRE: Gradient echo; MTX: Methotrexate.
}

\section{Authors' contributions}

MA, AY, YM and HA helped in data collection and statistical analysis. MA, AY and $H A$ reviewed all patients' $M R I$ images. MA analyzed and interpreted the patient data. MA wrote the manuscript. All authors read and approved the final manuscript.

\section{Funding}

Not applicable (no funding was provided).

\section{Availability of data and materials}

The datasets used and/or analyzed during the current study are available from the corresponding author on reasonable request.

\section{Declarations}

\section{Ethics approval and consent to participate}

Institutional (Faculty of Medicine 'Kasr El-Ainy Hospital' and National Cancer Institute, Cairo University) ethical clearance was taken before conducting this prospective study; March 2017-Reference number: not available. Written consent was obtained from patients or their authorized representatives.

\section{Consent for publication}

All patients included in this research gave written informed consent to publish the data contained within this study. Our patients were less than 17 years old, and when consent for publication was requested, written informed consent for the publication of these data was given by their parent or legal guardian.

\section{Competing interests}

The authors declare that they have no competing interests.

\section{Author details}

${ }^{1}$ Department of Diagnostic and Interventional Radiology, National Cancer Institute, Cairo University, 1 Kasr Elainy Street Fom Elkalig, Cairo, Egypt.

${ }^{2}$ Department of Diagnostic and Interventional Radiology, Faculty of Medicine, Cairo University, 12 El Manial Street, El Kasr Elainy, Cairo, Egypt. ${ }^{3}$ Department of Pediatric Oncology, National Cancer Institute, Cairo University, 1 Kasr Elainy Street Fom Elkalig, Cairo, Egypt.

Received: 6 May 2021 Accepted: 11 September 2021

Published online: 24 September 2021

\section{References}

1. Siegel RL, Miller KD, Jemal A (2015) Cancer statistics, 2015. CA Cancer J Clin 65(1):5-29

2. Ward E, DeSantis C, Robbins A et al (2014) Childhood and adolescent cancer statistics, 2014. CA Cancer J Clin 64(2):83-103

3. Rossi A, Morana G, Gandolfo C et al (2010) Neuroradiology of chemotherapeutic neurotoxicity in children. Neuroradiol J 23(2):183-190

4. Espagnet MCR, Pasquini L, Napolitano A et al (2017) Magnetic resonance imaging patterns of treatment-related toxicity in the pediatric brain: an update and review of the literature. Pediatr Radiol 47(6):633-648

5. Vázquez E, Delgado I, Sanchez-Montanez A et al (2011) Side effects of oncologic therapies in the pediatric central nervous system: update on neuroimaging findings. Radiographics 31(4):1123-1139

6. Psimaras D, Leclercq D, Ricard D et al (2014) Central nervous system complications in patients undergoing chemotherapy. In: Imaging of complications and toxicity following tumor therapy. Springer, Cham, pp 61-92

7. Torrisi JM, Schwartz LH, Gollub MJ et al (2011) CT findings of chemotherapy-induced toxicity: what radiologists need to know about the clinical and radiologic manifestations of chemotherapy toxicity 1. Radiology 258(1):41-56

8. Granata G, Greco A, lannella G et al (2015) Posterior reversible encephalopathy syndrome-Insight into pathogenesis, clinical variants, and treatment approaches. Autoimmun Rev 14(9):830-836 
9. Aracki-Trenkić A, Stojanov D, Trenkić M et al (2016) Imaging characteristics of posterior reversible encephalopathy syndrome (Pres). Acta Med Med 55(1):64-69

10. Siebert E, Spors B, Bohner G et al (2013) Posterior reversible encephalopathy syndrome in children: radiological and clinical findings-a retrospective analysis of a German tertiary care center. Eur J Pediatr Neurol 17:169-175

11. Alvis-Miranda HR, Castellar-Leones SM, Alcala-Cerra G (2013) Cerebral sinus venous thrombosis. J Neurosci Rural Pract 4(4):427-438

12. Kontzialis M, Huisman TAGM (2018) Toxic-metabolic neurologic disorders in children: a neuroimaging review. J Neuroimaging 28:587-595

13. Kamiya-Matsuoka C, Paker AM, Chi L et al (2016) Posterior reversible encephalopathy syndrome in cancer patients: a single institution retrospective study. J Neuro-Oncol 128(1):75-84

14. Khan RB, Sadighi ZS, Zabrowski J et al (2016) Imaging patterns and outcome of posterior reversible encephalopathy syndrome during childhood cancer treatment. Pediatr Blood Cancer 63(3):523-526

15. Wagih A, Mohsen L, Rayan MM et al (2015) Posterior reversible encephalopathy syndrome (PRES): restricted diffusion does not necessarily mean irreversibility. Pol J Radiol 80:210-216

16. Bidar F, Faeghi F, Ghorbani A (2016) Assessment of cerebral venous sinus thrombosis using $T 2 *$-weighted gradient echo magnetic resonance imaging sequences. Iran J Neurol 15(2):96-99
17. Wani NA, Kosar T, Pala NA et al (2010) Sagittal sinus thrombosis due to L-asparaginase. J Pediatr Neurosci 5(1):32

18. Al-said Y, Gulab S, Bayoumi M et al (2013) Cerebral sinus venous thrombosis due to asparaginase therapy. Case Rep Hematol 2013:5

19. El Damarawy EAA, El-Nekiedy AM, Fathi AM et al (2012) Role of magnetic resonance venography in evaluation of cerebral veins and sinuses occlusion. Alex J Med 48(1):29-34

20. Choi JE, Weon YC, Park GM et al (2018) Comparison of MRI sequences for the detection of cerebral venous sinus thrombosis during follow-up examination. J Korean Soc Radiol 78(5):330-339

21. Claire G, Patricia F, Patricia L, e t al, (2020) Methotrexate-induced acute myelopathy in a teenager with high-risk acute lymphoblastic leukemia. J Pediatr Hematol Oncol 42(4):e262-e264

22. Youssef AA, Raafat TA, Madney Y (2015) Child with acute methotrexate related neurotoxicity: Can diffusion weighted MRI help?? Egypt J Radiol Nucl Med 46(4):1149-1153

\section{Publisher's Note}

Springer Nature remains neutral with regard to jurisdictional claims in published maps and institutional affiliations.

\section{Submit your manuscript to a SpringerOpen ${ }^{\circ}$ journal and benefit from:}

- Convenient online submission

- Rigorous peer review

- Open access: articles freely available online

- High visibility within the field

Retaining the copyright to your article

Submit your next manuscript at $\gg$ springeropen.com 